\title{
NUTRIGENOMICS AS A TOOL IN THE PREVENTION OF LIPOTOXICITY: THE CASE OF SOY PROTEIN
}

\author{
Nimbe Torres, IVÁn Torre-Villalvazo and Armando R. Tovar* \\ Department of Nutrition Physiology, Instituto Nacional de Ciencias Médicas y Nutrición Salvador Zubirán, \\ Mexico City, Mexico.
}

\begin{abstract}
Obesity is associated with an increase of several metabolic disorders leading to the development of diseases such as type 2 diabetes and cardiovascular disease. This is due in part to the ectopic accumulation of triglycerides in organs that are nonadipose tissues, leading to lipotoxicity. Particularly, in the liver, the accumulation of lipids, mainly of triglycerides, leads to the formation of fatty liver. The accumulation of lipids in skeletal muscle and pancreas associates with insulin resistance and a decrease in insulin secretion, respectively. In addition, it has been suggested that dysbiosis of the gut microbiota can contribute to the process of lipid accumulation in non-adipose tissues, especially in the liver. The aim of the present review is to highlight the mechanisms associated with the development of lipotoxicity, and how with the advances in nutrigenomics, it is now possible to understand the molecular mechanisms by which some nutrients can attenuate the ectopic accumulation of triglycerides in non-adipose tissues. Particularly, we emphasize research conducted on the molecular mechanisms of action of soy protein and some of its isoflavones, and how these can reduce lipotoxicity by preventing the accumulation of lipids in the liver, skeletal muscle, and pancreas, as well as their role on the gut microbiota to attenuate the development of fatty liver. Thus, nutrigenomics is opening new dietary strategies based on several functional foods that can be used to ameliorate the pathologies associated with lipotoxicity. (REV INVEST CLIN. 2019;71:157-67)
\end{abstract}

Key words: Lipotoxicity. Fatty liver. Insulin resistance. Adipose tissue. Gut microbiota. Soy protein. Isoflavones.

Corresponding author:

*Armando R. Tovar

Department of Fisiología de la Nutrición

Instituto Nacional de Ciencias Médicas

y Nutrición Salvador Zubirán

Av. Vasco de Quiroga, 15

Col. Belisario Domínguez Sección XVI, Del. Tlalpan $\quad$ Received for publication: 4-12-2018

C.P. 14080, Mexico City, Mexico

E-mail: tovar.ar@gmail.com

Approved for publication: 21-12-2018

DOI: $10.24875 / R I C .18002843$ 


\section{INTRODUCTION}

Although several pharmacological strategies have been used for the treatment of accumulation of lipids particularly in the liver as occurs in non-alcoholic fatty liver disease (NAFLD), there are now various dietary treatments that can contribute to attenuate this process ${ }^{1}$. To this end, nutrigenomics, which studies how nutrients can regulate the expression of genes involved in metabolic pathways, allows investigating whether specific nutrients may be beneficial in the prevention of certain metabolic diseases, including the excessive accumulation of lipids in several organs or tissues, to prevent lipotoxicity ${ }^{2}$. Nutrients can influence the activity of certain transcription factors, which control the expression of genes involved in specific metabolic pathways, such as lipogenesis, fatty acid oxidation, and lipid mobilization, among others ${ }^{3}$. In addition, nutrients can also modulate the expression of genes indirectly through the regulation of the secretion of hormones that can activate transcription factors by specific signaling pathways ${ }^{4}$. Both direct and indirect mechanisms converge in the transcriptional regulation of specific genes, which can modify the accumulation of lipids in the tissues. This effect can be enhanced since nutrients can also modulate the gene expression pathways at the translation or at the post-translational level ${ }^{5}$. As a consequence, there is a reduction of lipotoxicity in different organs. Therefore, the aim of the present review is to highlight the mechanisms associated with the development of lipotoxicity, and how with the advances in nutrigenomics, it is now possible to understand the molecular mechanisms by which some nutrients can attenuate the ectopic accumulation of triglycerides in non-adipose tissues, leading to the elaboration of new dietary strategies to prevent or attenuate tissue lipotoxicity. In particular, several studies using soy protein and some of its isoflavones have demonstrated that this concept can be successfully used in preclinical and clinical research ${ }^{6-9}$.

\section{LIPOTOXICITY}

\section{Adipose tissue expandability and metabolic health}

Adipose tissue plays a key role in whole-body energy homeostasis by storing excess dietary energy as triglycerides during feeding, and releasing free fatty acids to provide energy substrates during fasting, stress, or physical activity ${ }^{10}$. In addition, the mobilization of fatty acids is accompanied by an increase in the secretion of leptin by the adipose tissue. The purpose of the increase in the amount of leptin released by adipose tissue is to stimulate the oxidation of fatty acids that have accumulated in non-adipose tissues $^{11,12}$. Adipose tissue mass is dynamically regulated by energy intake and expenditure ${ }^{13}$. During periods of increased energy intake, adipose tissue expands by both hypertrophy and hyperplasia to provide sufficient adipocytes for the efficient storage of triglycerides ${ }^{14}$. The main mediator of adipose tissue metabolism is the peroxisome proliferator-activated receptor (PPAR) $\gamma$, a ligand-activated transcription factor recognized as the "master regulator" of adipose tissue function ${ }^{15,16}$. PPAR $\gamma$ controls the transcriptional program of adipocyte differentiation, lipid synthesis, triglycerides esterification, lipid droplet formation, and adipokine secretion ${ }^{17}$.

Observations in animal models with altered adipose tissue function have revealed the pivotal role of adipose tissue in metabolic homeostasis ${ }^{18}$. Transgenic mice unable to recruit sufficient adipose tissue (lipodystrophic mice) develop a renal injury, hepatic steatosis, and whole-body insulin resistance ${ }^{19}$. When lipodystrophic mice are fed a high-fat diet, they do not become obese compared to wild-type mice. However, they develop type 2 diabetes many weeks earlier than their obese wild-type littermates ${ }^{20}$. Conversely, mice with increased capacity for adipose tissue recruitment become massively obese when fed a high-fat diet but are resistant to develop metabolic syndrome ${ }^{21}$. The divergent metabolic response of these mice to a highfat diet relies on the function of their adipose tissue. Adipose tissue is known to have a limited capacity to accumulate triglycerides and this will depend on its capacity of expansion 22 . However, the "expandability" of adipose tissue is different for every individual. Thus, subjects with high capacity to expand their adipose tissue will manage better the chronic consumption of food energy than those with a limited capacity for expansion. These latter subjects quickly showed abnormalities of metabolic syndrome such as hyperinsulinemia and hyperlipidemia. When adipose tissue expansion reaches its maximum capacity, adipocytes become dysfunctional, and various metabolic consequences appear in these subjects ${ }^{23,24}$. The 
impaired ability of the adipose tissue to expand during chronic overnutrition is mediated by reduced activity of PPAR $\gamma$. The mechanisms leading to PPAR $\gamma$ impairment during obesity are in part mediated by local pro-inflammatory signaling, leading to nuclear factor $\mathrm{\kappa B}$, and Jun- $\mathrm{N}$-terminal kinase activation and translocation to the nucleus. As a consequence, there is a reduction in PPAR $\gamma$ activity, impairing adipose tissue function ${ }^{25}$.

\section{Lipotoxicity as a common mediator in organ damage}

The unrestrained release of fatty acids from adipose tissue during obesity increases their uptake in nonadipose tissues, leading to cellular damage and organ dysfunction, and a process named lipotoxicity ${ }^{23}$. Lipotoxicity occurs when an inordinate amount of fatty acids accumulates in cells in a magnitude that surpasses the oxidative capacity of the cell to utilize the excess of fatty acids. Fatty acids are then esterified leading to the accumulation of triglycerides, as well as the formation of other lipids such as ceramides and diacylglycerides. The accumulation of these lipid species causes serious metabolic damage in various organs, mainly the liver, pancreas, and heart ${ }^{26}$.

Recent evidence has demonstrated that endoplasmic reticulum (ER) stress is also involved in lipotoxic cell dysfunction and death ${ }^{27}$. The ER is the organelle responsible for the synthesis, maturation and trafficking of secretory, and membrane-associated proteins. During acute ER perturbations, activation of the unfolded protein response (UPR) mitigates ER stress by reducing protein synthesis, facilitating protein degradation, and increasing production of protein folding chaperones. However, when ER stress is excessive, prolonged, or insufficiently neutralized, the UPR activates inflammatory and apoptotic pathways ${ }^{28}$. Several basic and clinical studies have observed that during obesity, ER stress occurs as a result of the accumulation of triglycerides in nonadipose tissues, leading to lipotoxicity in the liver, skeletal muscle, heart, and pancreas, among other organs (Fig. 1).

During feeding periods, the circulating glucose and lipids are uptake and stored in the liver to form glycogen and triglycerides, respectively. Some of these functions are coordinated by the transcription factors sterol regulatory element-binding protein-1 (SREBP-1) $)^{29}$ and carbohydrate-responsive elementbinding protein ${ }^{30}$. SREBP-1 is activated by insulin and induces the expression of lipogenic genes ${ }^{31}$, increasing fatty acid synthesis from glucose ${ }^{32}$. However, in obese insulin-resistant subjects, the persistent hyperinsulinemia maintains lipogenesis 5 -fold higher than in healthy subjects, even during fasting ${ }^{33}$, and leading to triglyceride accumulation ${ }^{34}$. In addition to hepatic lipogenesis, the increased flow of fatty acids from adipose tissue to the liver exacerbates the accumulation of triglycerides, resulting in the development of hepatic steatosis or NAFLD, which is highly prevalent in obese subjects ${ }^{35}$.

In addition, the metabolic fate of glucose and fatty acids in skeletal and cardiac muscle is under the control of the Randle cycle, in which glucose utilization inhibits fatty acid oxidation and vice versa ${ }^{36}$. Thus, in obesity, the sustained fatty acid release from hypertrophic adipose tissue provides a continuous supply of fatty acids to skeletal muscle or heart, impairing glucose metabolism, and leading to metabolic inflexibility ${ }^{37}$. The metabolic inflexibility in skeletal muscle also reduces insulin-stimulated glucose uptake. The elevation of circulating glucose stimulates pancreatic beta cells to increase the release of insulin resulting in hyperinsulinemia ${ }^{38}$. To restore metabolic flexibility, the skeletal muscle increases mitochondrial fatty acid oxidation through enhanced mitochondrial abundance and/or activity mediated by PPAR $\delta$ and by the peroxisome proliferator-activated receptor gamma coactivator 1-alpha ${ }^{39,40}$, which is activated by the enzyme AMP-activated protein kinase (AMPK) ${ }^{41}$. Leptin signaling also stimulates fatty acid oxidation and glucose uptake in skeletal muscle by activating $\mathrm{AMPK}^{42}$. However, lipotoxicity elicits ER stress in skeletal muscle, which, in turn, induces leptin resistance in muscle cells ${ }^{43}$. As a result, the mitochondrial betaoxidation of lipids is reduced and lipid accumulation increases even further.

During obesity, the high flow of circulating levels of fatty acids from adipose tissue is also accumulated in the pancreas generating lipotoxicity ${ }^{44}$. It has been demonstrated that the excess fatty acids stimulate the synthesis of ceramides and ER stress, causing the death of pancreatic beta cells. As a consequence, there is a reduction in the beta-cell mass leading to 
Figure 1. Development of lipotoxicity by the chronic ingestion of a high-energy diet. The adipose tissue is capable of storing the excess of energy in the form of triglycerides. At some stage, adipocytes become dysfunctional, leading to an elevated release of free fatty acids (FFA). The excess FFA are uptake by non-adipose tissues, including the liver, the skeletal muscle, and the pancreas among others, leading to lipotoxicity in different organs. The dysbiosis of the gut microbiota can enhance these effects.

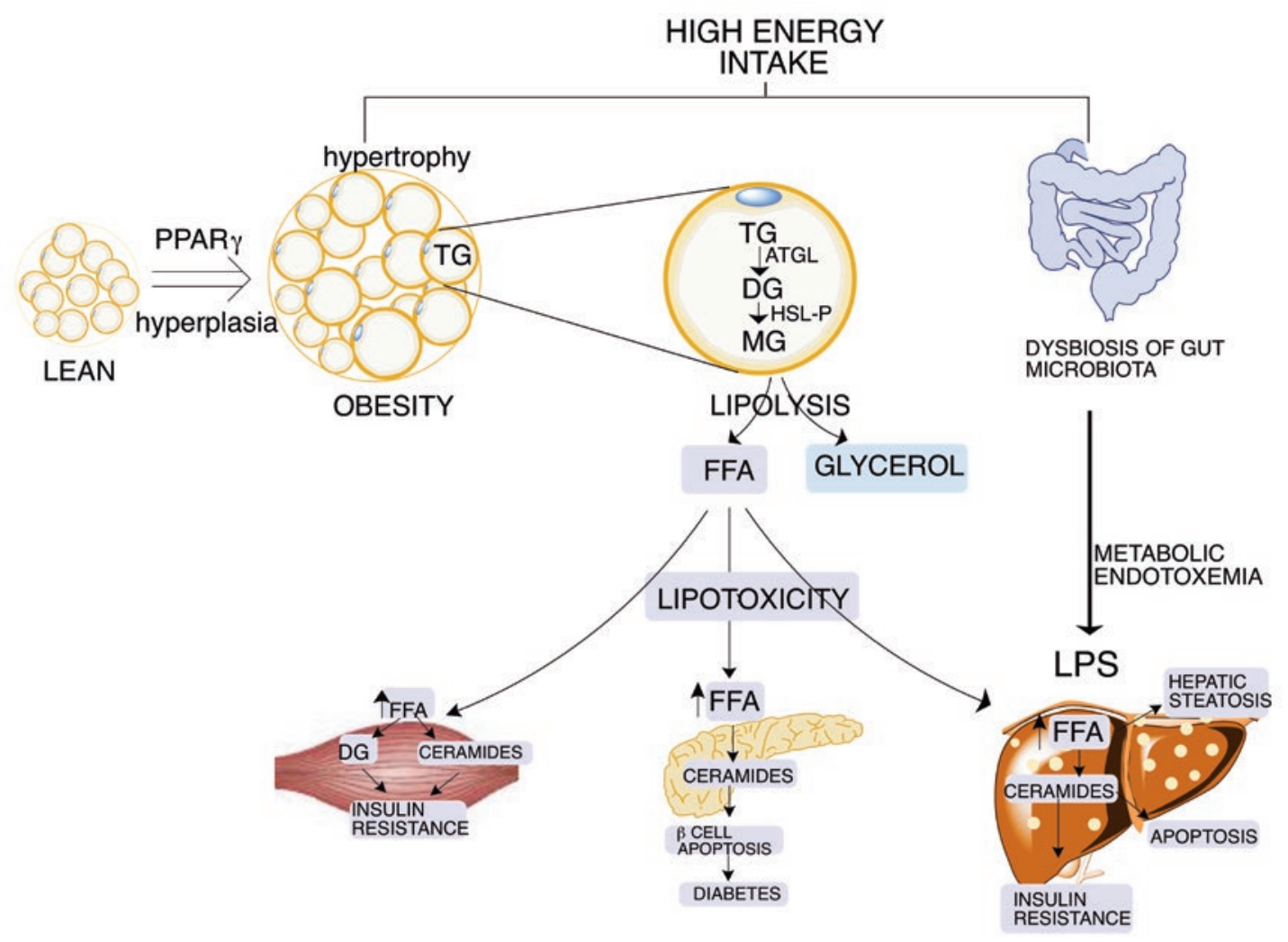

a decrease in insulin synthesis resulting in hyperglycemia, which in the long-term triggers the appearance of type-2 diabetes ${ }^{45}$.

\section{Lipotoxicity and the gut microbiota: dysbiosis and metabolic endotoxemia}

The gut microbiota is composed of trillions of microorganisms, and their collective genomes contain > 150 times more genes than the human genome ${ }^{46}$. Studies in animal models and humans have documented a distinct composition of the gut microbiota between lean and obese subjects ${ }^{47}$. The alteration in the taxonomic proportion of the bacterial population of the gut microbiota is a condition named dysbiosis ${ }^{48}$. A gut microbiota with dysbiosis is characterized by increasing the energy harvest from the diet, fat storage, alteration of satiety signals in the brain, impairment in cholesterol and bile acid metabolism, and induction of low-grade systemic inflammation also called "metabolic endotoxemia." 49

Recently, it has been demonstrated that alterations in microbial communities impact liver function. An increase in pathogenic bacteria in the intestinal lumen reduces gut barrier function, leading to an increase in intestinal permeability. The reduction in enterocyte tight junctions allows bacteria to reach the portal circulation and liver. Bacteria and bacterial-derived lipopolysaccharide bind to toll-like receptor, activating pro-inflammatory signaling in the liver, leading to the progression of NAFLD ${ }^{50}$. In addition, it is known that the accumulation of lipids in the liver is associated with dysbiosis of the gut microbiota, especially with 
a reduction of Faecalibacterium prausnitzii and an increase in Enterobacteria ${ }^{51}$.

\section{NUTRIGENOMICS}

There is evidence that several functional foods or dietary bioactive compounds can attenuate lipotoxicity ${ }^{52-58}$. One extensively studied food is soybean, particularly soy protein. Soybean is a legume that is widely consumed worldwide. It has been established that from $1961 \%$ to $2014,75 \%$ and $90 \%$ of the cultivated area under legumes was allocated to soybean (Glycine max) in South America and North America, respectively. In Asia, $76 \%$ of the area cultivated under legumes was allocated to four species (i.e., soybean, beans, groundnut, and chickpea). The composition of soybean has been extensively studied, and it contains between $36 \%$ and $40 \%$ of the protein in which the most abundant proteins are glycinin and $\beta$-conglycinin. In addition, soybean contains around $20 \%$ of lipids as oil, between $30 \%$ and $35 \%$ of carbohydrates, and some important minor constituents such as phytic acid and isoflavones ${ }^{59,60}$.

Soy protein has been considered a high-quality protein according to the assessment of the protein digestibility-corrected amino acid score (PDCAAS). Soy protein, after correcting for digestibility, provides amino acids equal to or in excess of requirements and receives a PDCAAS of 1 , indicating that it can meet the protein needs of infants and adults when consumed as the only source of protein at the recommended level of protein intake.

Some studies have shown that the consumption of soy protein can be used as part of the dietary strategy for the treatment of several pathological conditions, including renal disease ${ }^{62-65}$ and elevated concentration of low-density lipoproteins (LDL)cholesterol ${ }^{66,67}$. In addition, the use of soy protein has been shown to attenuate the development of lipotoxicity in several organs, as described below.

\section{Soy protein regulates lipid metabolism in the liver by transcriptional mechanisms decreasing lipotoxicity}

In the last decade, several studies have reported how certain nutrients can modulate the expression of genes involved in the accumulation of triglycerides in the liver, as is the case of soy protein ${ }^{68}$ (Fig. 2). It has been shown that soy protein can prevent the liver accumulation of triglycerides, despite the consumption of a high-fat diet. The mechanism by which soy protein regulates the accumulation of triglycerides involves the modulation of SREBP-1 activity ${ }^{69,70}$. The activation of SREBP-1 is stimulated by insulin and reduced by glucagon; therefore, the insulin/glucagon ratio determines the SREBP-1 expression and activity $^{71}$.

Some studies have shown that the increase in serum insulin produces a proportional increase in the concentration of the transcription factor SREBP-1 depending on the type of protein consumed. Rats fed a diet containing soy protein as the sole source of protein have a lower increase in serum insulin than rats fed the same percentage of a protein whose only source is casein. As a consequence, the liver of rats fed a soy protein diet expressed a low concentration of SREBP-1 mRNA and protein abundance of the mature form, as well as its target genes, such as the fatty acid synthase or the malic enzyme, among others. In contrast, the abundance of hepatic mRNA and protein abundance of the mature form of SREBP-1 were high, as well as of its target genes when rats were fed a casein diet ${ }^{69}$. This difference in the expression of SREBP-1 according to the type of protein consumed led to a lower accumulation of triglycerides and cholesterol in the liver of rats fed the soy protein diet, compared to rats fed the casein diet. These findings indicate that the type of protein consumed in the diet can modulate hepatic lipogenesis ${ }^{69}$. Interestingly, a similar outcome has been observed in Zucker fa/fa rats fed different types of proteins in the diet, despite the fact that these animals develop hyperinsulinemia. The liver of Zucker $\mathrm{fa} / f a$ rats fed a soy protein diet also show low levels of expression of SREBP-1 compared to rats fed a casein diet, resulting in a low accumulation of liver lipids, and mainly triglycerides ${ }^{72}$.

It has been shown that the consumption of soy protein decreases the serum concentration of insulin by reducing its secretion from pancreatic $\beta$ cells. Studies in rats fed soy protein using hyperglycemic clamps as well as in vitro pancreatic islet analysis have shown that the serum amino acid pattern generated after consumption of a soy protein diet, as well as the 
Figure 2. The consumption of soy protein and its isoflavones can ameliorate the lipotoxicity produced by the consumption of high-fat/carbohydrate diets. This effect occurs by improving the dysbiosis of the gut microbiota, by modifying pancreatic secretion of insulin and glucagon leading to a reduction in lipogenesis. In addition, adipocyte functionality improves these health benefits. Soy isoflavones can enhance fatty acid oxidation in skeletal muscle and increase white adipose tissue browning.

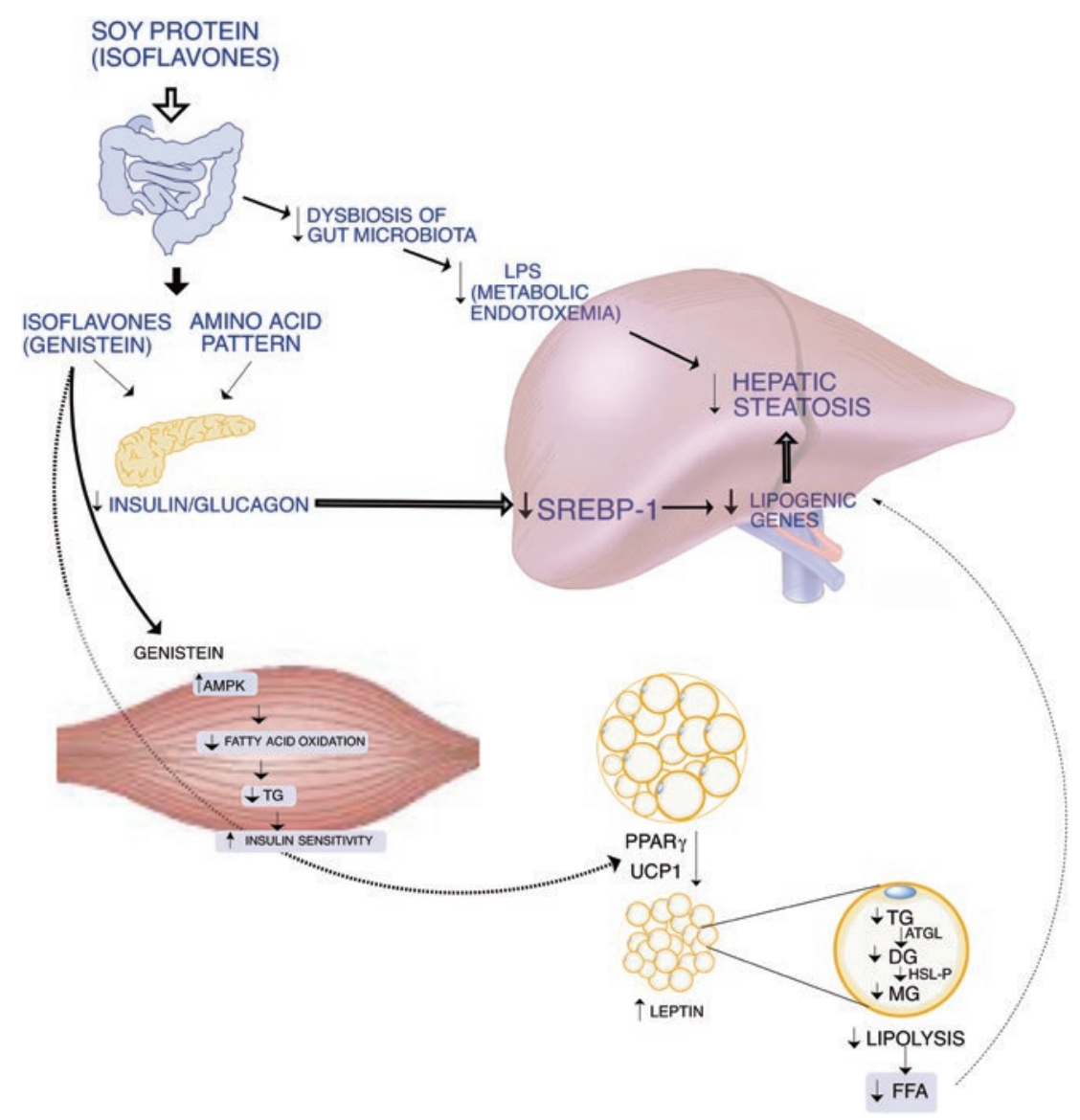

isoflavones present in soy protein, can decrease pancreatic secretion of insulin ${ }^{73}$. On the other hand, there is also evidence that ingestion of a soy protein diet stimulates the secretion of glucagon. Therefore, the consumption of soy protein reduces the insulin/glucagon ratio causing a low SREBP-1 expression. In fact, there is a linear correlation between the insulin/glucagon ratio and the abundance of SREBP-1 mRNA (Fig. 3). In addition, recent evidence shows that genistein is capable of inhibiting voltage-dependent Kv2 potassium channels, which may be involved in part in the decrease in insulin secretion stimulated by glucose ${ }^{74}$.

The decrease in hepatic lipogenesis due to the consumption of soy protein compared to animals fed with casein is accompanied by an increase in the oxidative capacity of fatty acids in the liver. It has been observed that in rats fed soy protein, there is an increase in the expression of the transcription factor PPAR $\alpha$, which is responsible for stimulating the expression of genes involved in fatty acid oxidation ${ }^{75,76}$. As a consequence, there is an increase in the expression of its target genes such as carnitine palmitoyltransferase- 1 , an enzyme that facilitates the entry of fatty acids into the mitochondria to be catabolized through $\beta$-oxidation ${ }^{72}$. Thus, the consumption of soy protein favors a balance of a decrease in hepatic lipogenesis and an increase in the oxidation of fatty acids, leading to a low accumulation of triglycerides in the liver, and preventing lipotoxicity. In fact, it has been demonstrated that soy protein reduces hepatic steatosis in Zucker rats ${ }^{77}$. 
Although few studies have been conducted in humans, there is evidence in subjects with NAFLD, that consumption of a diet containing soy protein can reduce the levels of alanine- and aspartate-aminotransferases, as well of serum fibrinogen, suggesting a beneficial effect in these patients ${ }^{78}$. However, longterm studies are needed to evaluate the impact of soy protein in subjects with NAFLD, particularly assessing hepatic lipid accumulation. Nonetheless, a recommended dietary strategy for NAFLD is a reduction in energy intake accompanied by the inclusion of omega- 3 fatty acids and soy protein in the diet ${ }^{1}$.

\section{The soy protein-associated isoflavone genistein prevents skeletal muscle lipotoxicity by increasing fatty acid oxidation}

It has been demonstrated that the reduction in the accumulation of lipids in the skeletal muscle is associated with an improvement of insulin sensitivity ${ }^{79}$. This condition is mediated in part by the activation of AMPK leading to acetyl-CoA carboxylase 1 inactivation, possibly together with malonyl-CoA decarboxylase activation, resulting in a decrease of malonyl-CoA concentration that favors fatty acid oxidation. In addition, another function of AMPK in skeletal muscle is to stimulate GLUT4 translocation to the plasma membrane, which increases glucose uptake and metabolism ${ }^{80}$. Thus, the insulin-independent stimulation of glucose transport accompanied by an increase in fatty oxidation in muscle and heart, indicate that AMPK is a key target for pharmacological therapies in insulin-resistant subjects ${ }^{80}$. It has been described that some bioactive food compounds such as isoflavones and resveratrol can activate AMPK, stimulating glucose uptake and fatty acid oxidation in skeletal muscle. Indeed, consumption of genistein increases insulin sensitivity in obese mice associated with an increase in AMPK activity. This is associated with an increase in fatty acid oxidation in skeletal muscle, also demonstrated using $\mathrm{C} 2 \mathrm{C} 12$ myotubes $^{81}$ (Fig. 2).

Recent meta-analyses have shown that consumption of soy products and particularly soy protein in humans can be associated with a lower risk of type 2 diabetes ${ }^{82-84}$. However, there is heterogeneity of outcomes between many investigations, possibly
Figure 3. Association between the insulin/glucagon ratio and the concentration of sterol regulatory element-binding protein-1 (SREBP-1) mRNA in rats fed soy protein-based diets containing different percentages of fat. Male obese Zucker $(\mathrm{fa} / \mathrm{fa})$ rats had free access to the experimental diets for 2 months containing $5 \%$ soybean oil, $10 \%$ soybean oil or coconut oil, and $20 \%$ soy protein as the protein source. Serum insulin and glucagon concentration were determined by ELISA and by radioimmunoassay. Total RNA from liver samples was isolated, and mRNA levels of SREBP-1 were measured by real-time quantitative polymerase chain reaction in triplicate. HPRT mRNA was used as an invariant control.

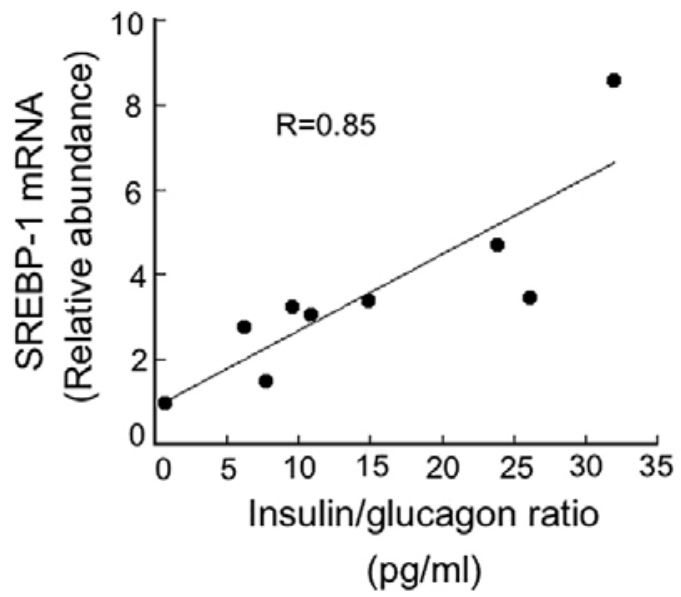

associated with the population studied, the type of soy foods administered in the trial, and the presence of isoflavones. Nonetheless, most of the studies show an improvement in fasting glucose or insulin concentrations or amelioration of insulin sensitivity ${ }^{85}$. In addition, this evidence is in agreement with a meta-analysis study showing that the consumption of soy isoflavones can reduce fasting glucose and fasting insulin in postmenopausal women ${ }^{86}$. Further studies are needed to confirm the effect of isoflavones on glucose metabolism in humans.

\section{Soy protein prevents adipose tissue dysfunction}

Dysfunction of the adipose tissue is evident when adipocytes have a low capacity to store the excess of energy as triglycerides ${ }^{22}$. Previous studies show that body weight gain is reduced when rats are fed a high-fat diet containing soy protein, compared to those fed a high-fat diet containing casein as a protein source. The difference in body weight between groups fed with these diets is due to a lower amount 
of body fat in animals fed a soy protein diet ${ }^{71}$. Further studies have even shown that consumption of soy protein stimulates the number of adipocytes of smaller size, containing small lipid droplets, indicative of functional adipocytes. Thus, the concentration of circulating leptin, an adipokine secreted by the adipocytes, is lower in rats fed soy protein than in those fed casein. This process is magnified because the consumption of the type of dietary protein alters the secretion and sensitivity of the organism to leptin. This adipokine has different functions, including the hypothalamic control of food intake, in which soy protein increases the expression of the leptin receptor in the hypothalamus $^{71}$. Furthermore, leptin stimulates in peripheral tissues such as liver and skeletal muscle, among others, fatty acid oxidation through the activation of $\mathrm{AMPK}^{87}$ and the transcription factor PPAR ${ }^{88}$. Studies in rats fed high-fat casein diets reveal the development of leptin resistance leading to hyperleptinemia, while rats fed soy protein show normoleptinemia indicative of normal sensitivity to the action of leptin ${ }^{71}$.

This evidence suggests that soy protein can increase the functionality of adipose tissue. Evaluation by microarray analysis in rats revealed that the consumption of soy protein modified the expression of 90 genes in adipose tissue compared with those fed a casein diet. These included genes coding for adipokines, members of the renin-angiotensin system, inflammation, and cell signaling. In addition, genes of lipid and carbohydrate metabolism were also modified, suggesting that soy protein is involved in an improvement of adipose tissue function ${ }^{89-91}$. Interestingly, one gene that is upregulated by the consumption of soy protein is PPAR $\gamma$.

The reduction in the expression of PPAR $\gamma$ in adipose tissue during obesity leads to a decrease in the ability of adipocytes to differentiate, esterify TG, and to release adipokines ${ }^{22,92}$. It is known that several PPAR $\gamma$ agonists can stimulate adipose tissue functionality, increasing its capacity of expansion leading to an improvement of glucose and lipid metabolism that favors the reduction of lipotoxicity in non-adipose tissues ${ }^{93}$. Thus, the increase of PPAR $\gamma$ expression in adipose tissue by soy protein is associated with an enhancement of adipose tissue functionality.
Recent evidence suggests that another mechanism that can be associated with an increase in adipose tissue functionality is the effect of soy protein to increase energy expenditure. This effect is mediated by the capacity of one of the soy protein isoflavones, genistein, to stimulate browning of white adipose tissue $^{94}$. Experimental evidence in cells and animal models have demonstrated that genistein can stimulate in adipose tissue the expression of the uncoupling protein 1, a protein involved in thermogenesis, as well as some of the browning markers ${ }^{94}$. Therefore, the increase of this process can stimulate the mitochondrial oxidation of fatty acids in the adipose tissue, which, in turn, can improve the functionality of the adipose tissue.

\section{Genistein modifies gut microbiota preventing metabolic endotoxemia}

Recent evidence has demonstrated that genistein can selectively modify the gut microbiota in mice fed a high-fat diet; an effect associated with a reduction in the insulin sensitivity compared to control mice fed a high-fat diet without genistein ${ }^{95}$. Interestingly, the modification in the gut microbiota was accompanied by a remarkable reduction of the circulating concentrations of LPS, preventing metabolic endotoxemia $^{95}$, a condition that has been associated with the development of NAFLD ${ }^{96}$. However, more studies are needed to determine whether the modification of the gut microbiota is directly associated with a reduction in hepatic lipotoxicity, despite the consumption of a high-fat diet.

\section{CONCLUSIONS}

Overall, this review summarizes the effects of soy protein-based on nutrigenomics studies that may contribute to develop new non-pharmacological strategies for patients with metabolic diseases associated with obesity, including lipotoxicity. Thus, soy protein can prevent or attenuate non-adipose tissue lipotoxicity directly by increasing fatty acid oxidation and indirectly by enhancing adipose tissue functionality. These effects are mediated by the regulation of key transcription factors involved in 
lipogenesis, fatty acid oxidation, and adipocyte differentiation. Nonetheless, further studies in humans are needed to determine if all subjects have the same response to these effects of soy protein. For instance, it has been demonstrated that some individuals are more susceptible to reduce total and LDL-cholesterol with a dietary portfolio of soy protein and soluble fiber depending on the presence of some gene variants ${ }^{97}$. Interestingly, the consumption of this dietary portfolio containing soy protein by hyperlipidemic subjects has a significant effect on increasing $\mathrm{HDL}$-cholesterol in subjects with genetic predisposition to have a low circulating concentration of $\mathrm{HDL}$-cholesterol, a condition highly prevalent in the Hispanic population ${ }^{98}$. Notably, a recent meta-analysis conducted on the health benefits of soy protein and its isoflavones in humans has demonstrated that their consumption is safe and has no adverse effects ${ }^{8}$. Nonetheless, further studies in the field of nutrigenomics and nutrigenetics are required to provide personalized nutritional advice in which soy protein may be a beneficial food component in patients with a risk of obesity-related lipotoxicity.

\section{REFERENCES}

1. Fan JG, Cao HX. Role of diet and nutritional management in non-alcoholic fatty liver disease. J Gastroenterol Hepatol. 2013; 28 Suppl 4:81-7.

2. Dongiovanni P, Valenti L. A nutrigenomic approach to non-alcoholic fatty liver disease. Int J Mol Sci. 2017;18:e1534

3. Ortega Á, Berná G, Rojas A, Martín F, Soria B. Gene-diet interactions in Type 2 diabetes: the chicken and egg debate. Int J Mol Sci. 2017;18:e1188

4. Goodridge AG, Fantozzi DA, Klautky SA, et al. Nutritional and hormonal regulation of genes for lipogenic enzymes. Proc Nutr Soc. 1991;50:115-22.

5. Berná G, Oliveras-López MJ, Jurado-Ruíz E, et al. Nutrigenetics and nutrigenomics insights into diabetes etiopathogenesis. Nutrients. 2014;6:5338-69

6. Kani AH, Alavian SM, Esmaillzadeh A, et al. Effects of a lowcalorie, low-carbohydrate soy containing diet on systemic inflammation among patients with nonalcoholic fatty liver disease: a parallel randomized clinical trial. Horm Metab Res. 2017; 49:687-92

7. Ma D, Taku K, Zhang $Y$, et al. Serum lipid-improving effect of soyabean $\beta$-conglycinin in hyperlipidaemic menopausal women. Br J Nutr. 2013;110:1680-4

8. Messina M. Soy and health update: evaluation of the clinical and epidemiologic literature. Nutrients. 2016;8:754.

9. Ruscica M, Pavanello C, Gandini S, et al. Effect of soy on metabolic syndrome and cardiovascular risk factors: a randomized controlled trial. Eur J Nutr. 2018;57:499-511.

10. Torres N, Torre-Villalvazo I, Tovar AR. Future directions in reducing hepatic lipotoxicity. Future Lipidol. 2006;1:331-41.

11. Unger $\mathrm{RH}$. Lipid overload and overflow: metabolic trauma and the metabolic syndrome. Trends Endocrinol Metab. 2003;14: 398-403.

12. Unger RH, Orci L. Diseases of liporegulation: new perspective on obesity and related disorders. FASEB J. 2001;15:312-21.
13. Hall KD, Heymsfield SB, Kemnitz JW, et al. Energy balance and its components: implications for body weight regulation. Am J Clin Nutr. 2012;95:989-94.

14. Virtue S, Vidal-Puig A. Adipose tissue expandability, lipotoxicity and the metabolic syndrome an allostatic perspective. Biochim Biophys Acta. 2010;1801:338-49.

15. Tontonoz P, Hu E, Graves RA, Budavari Al, Spiegelman BM. MPPAR gamma 2: tissue-specific regulator of an adipocyte enhancer. Genes Dev. 1994;8:1224-34.

16. Tontonoz P, Hu E, Spiegelman BM. Stimulation of adipogenesis in fibroblasts by PPAR gamma 2, a lipid-activated transcription factor. Cell. 1994;79:1147-56.

17. Lefterova MI, Haakonsson AK, Lazar MA, Mandrup S. PPAR $\gamma$ and the global map of adipogenesis and beyond. Trends Endocrinol Metab. 2014;25:293-302

18. Pellegrinelli V, Carobbio S, Vidal-Puig A. Adipose tissue plasticity: how fat depots respond differently to pathophysiological cues. Diabetologia. 2016;59:1075-88.

19. Liu XJ, Wu XY, Wang $H$, et al. Renal injury in seipin-deficient lipodystrophic mice and its reversal by adipose tissue transplantation or leptin administration alone: adipose tissue-kidney crosstalk. FASEB J. 2018;32:5550-62.

20. Kim JK, Gavrilova O, Chen Y, Reitman ML, Shulman GI. Mechanism of insulin resistance in A-ZIP/F-1 fatless mice. J Biol Chem 2000;275:8456-60.

21. Kim JY, van de Wall E, Laplante $M$, et al. Obesity-associated improvements in metabolic profile through expansion of adipose tissue. J Clin Invest. 2007;117:2621-37.

22. Vázquez-Vela ME, Torres N, Tovar AR. White adipose tissue as endocrine organ and its role in obesity. Arch Med Res. 2008; 39:715-28.

23. Carobbio S, Pellegrinelli V, Vidal-Puig A. Adipose tissue function and expandability as determinants of lipotoxicity and the metabolic syndrome. Adv Exp Med Biol. 2017;960:161-96.

24. Gray SL, Vidal-Puig AJ. Adipose tissue expandability in the maintenance of metabolic homeostasis. Nutr Rev. 2007 65:S7-12.

25. Baker RG, Hayden MS, Ghosh S. NF- $\kappa B$, inflammation, and metabolic disease. Cell Metab. 2011;13:11-22.

26. Unger RH, Clark GO, Scherer PE, Orci L. Lipid homeostasis, lipotoxicity and the metabolic syndrome. Biochim Biophys Acta. 2010;1801:209-14

27. Han J, Kaufman RJ. The role of ER stress in lipid metabolism and lipotoxicity. J Lipid Res. 2016;57:1329-38.

28. Hotamisligil GS. Endoplasmic reticulum stress and the inflammatory basis of metabolic disease. Cell. 2010;140:900-17.

29. Shimomura I, Bashmakov Y, Ikemoto S, et al. Insulin selectively increases SREBP-1C MRNA in the livers of rats with streptozotocin-induced diabetes. Proc Natl Acad Sci U S A. 1999:96:13656-61.

30. Linden AG, Li S, Choi HY, et al. Interplay between chREBP and SREBP-1c coordinates postprandial glycolysis and lipogenesis in livers of mice. J Lipid Res. 2018;59:475-87.

31. Horton JD, Goldstein JL, Brown MS. SREBPs: activators of the complete program of cholesterol and fatty acid synthesis in the liver. J Clin Invest. 2002;109:1125-31.

32. Xu X, So JS, Park JG, Lee AH. Transcriptional control of hepatic lipid metabolism by SREBP and chREBP. Semin Liver Dis. 2013; 33:301-11.

33. Schwarz JM, Linfoot P, Dare D, Aghajanian K. Hepatic de novo lipogenesis in normoinsulinemic and hyperinsulinemic subjects consuming high-fat, low-carbohydrate and low-fat, high-carbohydrate isoenergetic diets. Am J Clin Nutr. 2003;77:43-50.

34. Moon YA. The SCAP/SREBP pathway: a Mediator of hepatic steatosis. Endocrinol Metab (Seoul). 2017;32:6-10.

35. Dai W, Ye L, Liu A, et al. Prevalence of nonalcoholic fatty liver disease in patients with Type 2 diabetes mellitus: a meta-analysis. Medicine (Baltimore). 2017;96:e8179

36. Hue $L$, Taegtmeyer $H$. The randle cycle revisited: a new head for an old hat. Am J Physiol Endocrinol Metab. 2009;297:E578-91.

37. Soares RN, Reimer RA, Doyle-Baker PK, Murias JM. Metabolic inflexibility in individuals with obesity assessed by near-infrared spectroscopy. Diab Vasc Dis Res. 2017;14:502-9.

38. Jung $\mathrm{SH}$, Jung $\mathrm{CH}$, Reaven $\mathrm{GM}$, Kim SH. Adapting to insulin resistance in obesity: role of insulin secretion and clearance. Diabetologia. 2018;61:681-7

39. Fan W, Waizenegger W, Lin CS, et al. PPAR $\delta$ promotes running endurance by preserving glucose. Cell Metab. 2017;25:1186930000.

40. Kleiner S, Nguyen-Tran V, Baré O, et al. PPAR\{delta\} agonism activates fatty acid oxidation via PGC-1\{alpha\} but does not 
increase mitochondrial gene expression and function. J Biol Chem. 2009;284:18624-33.

41. Jäger S, Handschin C, St-Pierre J, Spiegelman BM. AMP-activated protein kinase (AMPK) action in skeletal muscle via direct phosphorylation of PGC-1alpha. Proc Natl Acad Sci U S A. 2007;104:12017-22.

42. Jensen J, O'Rahilly S. AMPK is required for exercise to enhance insulin sensitivity in skeletal muscles. Mol Metab. 2017;6:315-6.

43. Panzhinskiy E, Hua Y, Culver B, Ren J, Nair S. Endoplasmic reticulum stress upregulates protein tyrosine phosphatase $1 \mathrm{~B}$ and impairs glucose uptake in cultured myotubes. Diabetologia. 2013; 56:598-607.

44. Oh YS, Bae GD, Baek DJ, Park EY, Jun HS. Fatty acid-induced lipotoxicity in pancreatic beta-cells during development of Type 2 diabetes. Front Endocrinol (Lausanne). 2018;9:384.

45. Cnop M, Ladrière L, Igoillo-Esteve $M$, Moura RF, Cunha DA. Causes and cures for endoplasmic reticulum stress in lipotoxic $\beta$-cell dysfunction. Diabetes Obes Metab. 2010;12 Suppl 2:76-82.

46. Thursby E, Juge N. Introduction to the human gut microbiota. Biochem J. 2017;474:1823-36.

47. Baothman OA, Zamzami MA, Taher I, Abubaker J, Abu-Farha M. The role of gut microbiota in the development of obesity and diabetes. Lipids Health Dis. 2016;15:108.

48. Carding S, Verbeke K, Vipond DT, Corfe BM, Owen LJ. Dysbiosis of the gut microbiota in disease. Microb Ecol Health Dis. 2015; 26:26191.

49. Boulangé CL, Neves AL, Chilloux J, Nicholson JK, Dumas ME. Impact of the gut microbiota on inflammation, obesity, and metabolic disease. Genome Med. 2016;8:42.

50. Marra F, Svegliati-Baroni G. Lipotoxicity and the gut-liver axis in NASH pathogenesis. J Hepatol. 2018;68:280-95.

51. Munukka E, Pekkala S, Wiklund $P$, et al. Gut-adipose tissue axis in hepatic fat accumulation in humans. J Hepatol. 2014;61: 132-8.

52. Chavez-Santoscoy RA, Gutierrez-Uribe JA, Granados O, et al. Flavonoids and saponins extracted from black bean (Phaseolus vulgaris L.) seed coats modulate lipid metabolism and biliary cholesterol secretion in C57BL/6 mice. Br J Nutr. 2014; 112:886-99.

53. Chávez-Santoscoy RA, Tovar AR, Serna-Saldivar SO, Torres N, Gutiérrez-Uribe JA. Conjugated and free sterols from black bean (Phaseolus vulgaris L.) seed coats as cholesterol micelle disruptors and their effect on lipid metabolism and cholesterol transport in rat primary hepatocytes. Genes Nutr. 2014;9:367.

54. Leal-Díaz AM, Noriega LG, Torre-Villalvazo I, et al. Aguamiel concentrate from agave salmiana and its extracted saponins attenuated obesity and hepatic steatosis and increased akkermansia muciniphila in C57BL6 mice. Sci Rep. 2016:6:34242

55. Sanchez-Tapia M, Aguilar-Lopez M, Perez-Cruz C, et al. Nopal (Opuntia ficus indica) protects from metabolic endotoxemia by modifying gut microbiota in obese rats fed high fat/sucrose diet. Sci Rep. 2017;7:4716.

56. Serrano JC, Cassanye A, Martín-Gari M, Granado-Serrano AB, Portero-Otín M. Effect of dietary bioactive compounds on mitochondrial and metabolic flexibility. Diseases 2016;4:e14

57. Yan C, Sun W, Wang X, et al. Punicalagin attenuates palmitateinduced lipotoxicity in hepG 2 cells by activating the keap1nrf2 antioxidant defense system. Mol Nutr Food Res. 2016; 60:1139-49

58. Zhou Y, Lin S, Zhang L, Li Y. Resveratrol prevents renal lipotoxicity in high-fat diet-treated mouse model through regulating PPAR- $\alpha$ pathway. Mol Cell Biochem. 2016;411:143-50.

59. García MC, Torre M, Marina ML, Laborda F. Composition and characterization of soyabean and related products. Crit Rev Food Sci Nutr. 1997:37:361-91.

60. US Department of Agriculture, ARS. Nutrient Data Laboratory. USDA National Nutrient Database for Standard Reference. 2018; Available from: https://www.ndb.nal.usda.gov/ndb/ foods. [Visitado el 02-01-2019].

61. Torres NT, Tovar-Palacio AR. The importance of soy in Mexico, its nutritional value and effect on health. Salud Publica Mex. 2009:51:246-54.

62. Anderson JW. Beneficial effects of soy protein consumption for renal function. Asia Pac J Clin Nutr. 2008;17 Suppl 1:324-8.

63. Anderson JW, Blake JE, Turner J, Smith BM. Effects of soy protein on renal function and proteinuria in patients with Type 2 diabetes. Am J Clin Nutr. 1998;68:1347S-1353S.

64. Tovar AR, Murguía F, Cruz C, et al. A soy protein dietalters hepatic lipid metabolism gene expression and reduces serum lipids and renal fibrogenic cytokines in rats with chronic nephrotic syndrome. J Nutr. 2002;132:2562-9.

65. Trujillo J, Ramírez V, Pérez J, et al. Renal protection by a soy diet in obese zucker rats is associated with restoration of nitric oxide generation. Am J Physiol Renal Physiol. 2005;288:F108-16.

66. Jenkins DJ, Mirrahimi A, Srichaikul K, et al. Soy protein reduces serum cholesterol by both intrinsic and food displacement mechanisms. J Nutr. 2010;140:2302S-2311S

67. Li SS, Mejia SB, Lytvyn L, et al. Effect of plant protein on blood lipids: a systematic review and meta-analysis of randomized controlled trials. J Am Heart Assoc. 2017;6:e006659.

68. Torres N, Torre-Villalvazo I, Tovar AR. Regulation of lipid metabolism by soy protein and its implication in diseases mediated by lipid disorders. J Nutr Biochem. 2006;17:365-73.

69. Ascencio C, Torres N, Isoard-Acosta F, et al. Soy protein affects serum insulin and hepatic SREBP-1 mRNA and reduces fatty liver in rats. J Nutr. 2004;134:522-9.

70. Hashidume T, Sasaki T, Inoue J, Sato R. Consumption of soy protein isolate reduces hepatic SREBP-1c and lipogenic gene expression in wild-type mice, but not in FXR-deficient mice. Biosci Biotechnol Biochem. 2011;75:1702-7

71. Torre-Villalvazo I, Tovar AR, Ramos-Barragán VE, Cerbón-Cervantes MA, Torres N. Soy protein ameliorates metabolic abnormalities in liver and adipose tissue of rats fed a high fat diet. ] Nutr. 2008;138:462-8.

72. Tovar AR, Torre-Villalvazo I, Ochoa M, et al. Soy protein reduces hepatic lipotoxicity in hyperinsulinemic obese zucker fa/fa rats. J Lipid Res. 2005; 46:1823-32.

73. Noriega-López L, Tovar AR, Gonzalez-Granillo M, et al. Pancreatic insulin secretion in rats fed a soy protein high fat diet depends on the interaction between the amino acid pattern and isoflavones. J Biol Chem. 2007;282:20657-66.

74. Aréchiga-Figueroa IA, Morán-Zendejas R, Delgado-Ramírez M, Rodríguez-Menchaca AA. Phytochemicals genistein and capsaicin modulate kv2.1 channel gating. Pharmacol Rep. 2017; 69:1145-53.

75. Contreras AV, Torres N, Tovar AR. PPAR- $\alpha$ as a key nutritional and environmental sensor for metabolic adaptation. Adv Nutr. 2013;4:439-52.

76. Oliva ME, Chicco A, Lombardo YB. Mechanisms underlying the beneficial effect of soy protein in improving the metabolic abnormalities in the liver and skeletal muscle of dyslipemic insulin resistant rats. Eur J Nutr. 2015;54:407-19.

77. Hakkak R, Gauss CH, Bell A, Korourian S. Short-term soy protein isolate feeding prevents liver steatosis and reduces serum ALT and AST levels in obese female zucker rats. Biomedicines 2018;6:e55

78. Kani AH, Alavian SM, Esmaillzadeh A, Adibi P, Azadbakht L. Effects of a novel therapeutic diet on liver enzymes and coagulating factors in patients with non-alcoholic fatty liver disease: a parallel randomized trial. Nutrition. 2014;30:814-21.

79. Adams JM 2nd, Pratipanawatr T, Berria R, et al. Ceramide content is increased in skeletal muscle from obese insulin-resistant humans. Diabetes. 2004;53:25-31.

80. Thomson DM, Winder WW. AMP-activated protein kinase control of fat metabolism in skeletal muscle. Acta Physiol. (Oxf) 2009;196:147-54

81. Palacios-González B, Zarain-Herzberg A, Flores-Galicia I, et al. Genistein stimulates fatty acid oxidation in a leptin receptorindependent manner through the JAK2-mediated phosphorylation and activation of AMPK in skeletal muscle. Biochim Biophys Acta. 2014;1841:132-40.

82. Li W, Ruan W, Peng Y, Wang D. Soy and the risk of Type 2 diabetes mellitus: a systematic review and meta-analysis of observational studies. Diabetes Res Clin Pract. 2018;137:190-9.

83. Tian $\mathrm{S}, \mathrm{Xu} \mathrm{Q}$, Jiang $\mathrm{R}$, et al. Dietary protein consumption and the risk of Type 2 diabetes: a systematic review and meta-analysis of cohort studies. Nutrients. 2017;9:e982.

84. Tatsumi Y, Morimoto A, Deura K, et al. Effects of soybean product intake on fasting and postload hyperglycemia and Type 2 diabetes in japanese men with high body mass index: the saku study. J Diabetes Investig. 2013;4:626-33.

85. Ramdath DD, Padhi EM, Sarfaraz S, Renwick S, Duncan AM. Beyond the cholesterol-lowering effect of soy protein: a review of the effects of dietary soy and its constituents on risk factors for cardiovascular disease. Nutrients. 2017;9:e324.

86. Zhang YB, Chen WH, Guo JJ, et al. Soy isoflavone supplementation could reduce body weight and improve glucose metabolism in non-asian postmenopausal women a meta-analysis. Nutrition. 2013;29:8-14. 
87. Miranda N, Tovar AR, Palacios B, Torres N. AMPK as a cellular energy sensor and its function in the organism. Rev Invest Clin. 2007;59:458-69.

88. Alemán G, Torres N, Tovar AR. Peroxisome proliferator-activated receptors (PPARs) in obesity and insulin resistance development. Rev Invest Clin. 2004;56:351-67.

89. Díaz-Villaseñor A, Granados O, González-Palacios B, et al. Differential modulation of the functionality of white adipose tissue of obese zucker $(\mathrm{fa} / \mathrm{fa})$ rats by the type of protein and the amount and type of fat. J Nutr Biochem. 2013; 24:1798-809.

90. Frigolet ME, Torres N, Tovar AR. Soya protein attenuates abnormalities of the renin-angiotensin system in adipose tissue from obese rats. Br ] Nutr. 2012;107:36-44.

91. Frigolet ME, Torres N, Uribe-Figueroa L, et al. White adipose tissue genome wide-expression profiling and adipocyte metabolic functions after soy protein consumption in rats. J Nutr Biochem. 2011;22:118-29.

92. Spiegelman BM. PPAR-gamma: adipogenic regulator and thiazolidinedione receptor. Diabetes. 1998;47:507-14.

93. Medina-Gomez G, Gray S, Vidal-Puig A. Adipogenesis and lipotoxicity: role of peroxisome proliferator-activated receptor gamma (PPARgamma) and PPARgammacoactivator-1 (PGC1). Public Health Nutr. 2007;10:1132-7.

94. Aziz SA, Wakeling LA, Miwa S, et al. Metabolic programming of a beige adipocyte phenotype by genistein. Mol Nutr Food Res 2017;61:1600574

95. López P, Sánchez M, Perez-Cruz C, et al. Long-term genistein consumption modifies gut microbiota, improving glucose metabolism, metabolic endotoxemia, and cognitive function in mice fed a high-fat diet. Mol Nutr Food Res. 2018;62: e1800313.

96. Vespasiani-Gentilucci U, Gallo P, Picardi A. The role of intestinal microbiota in the pathogenesis of NAFLD: starting points for intervention. Arch Med Sci. 2018;14:701-6.

97. Guevara-Cruz M, Lai CQ, Richardson K, et al. Effect of a GFOD2 variant on responses in total and LDL cholesterol in mexican subjects with hypercholesterolemia after soy protein and soluble fiber supplementation. Gene. 2013;532:211-5.

98. Guevara-Cruz M, Tovar AR, Larrieta E, Canizales-Quinteros S, Torres $\mathrm{N}$. Increase in HDL-C concentration by a dietary portfolio with soy protein and soluble fiber is associated with the presence of the ABCA1R230C variant in hyperlipidemic mexican subjects. Mol Genet Metab. 2010;101:268-72. 